\title{
The Arabidopsis ISR1 Locus is Required for Rhizobacteria-Mediated Induced Systemic Resistance Against Different Pathogens
}

\author{
J. Ton ${ }^{1,2}$, J. A. Van Pelt ${ }^{1}$, L. C. Van Loon ${ }^{1}$, and C. M. J. Pieterse ${ }^{1}$ \\ 1 Phytopathology, Faculty of Biology, Utrecht University, Graduate School of Experimental Plant Sciences, Utrecht, The Netherlands \\ ${ }_{2}^{2}$ Present address: Laboratory of Biochemistry, NCCR, University of Neuchâtel, Rue Emile-Argand 9, 2007 Neuchâtel 7, Switzerland
}

Received: July 25, 2001; Accepted: January 17, 2002

\begin{abstract}
In Arabidopsis thaliana, non-pathogenic, root-colonizing Pseudomonas fluorescens WCS417r bacteria trigger an induced systemic resistance (ISR) that is phenotypically similar to pathogen-induced systemic acquired resistance (SAR). In contrast to SAR, WCS417r-mediated ISR is controlled by a salicylic acid (SA)-independent signalling pathway that requires an intact response to the plant hormones jasmonic acid (JA) and ethylene (ET). Arabidopsis accessions RLD1 and Ws-0 fail to express ISR against Pseudomonas syringae pv. tomato and show enhanced disease susceptibility to this pathogen. Genetic analysis of progeny from crosses between WCS417r-responsive and nonresponsive accessions demonstrated that ISR inducibility and basal resistance against $P$. syringae pv. tomato are controlled by a single dominant locus (ISR1) on chromosome III (Ton et al., $\left.1999^{[8]}\right)$. Here, we investigated the role of the ISR1 locus in ISR, SAR and basal resistance against three additional pathogens: Xanthomonas campestris pv. armoraciae, Peronospora parasitica and turnip crinkle virus (TCV), using accessions Col-0 (ISR1), RLD1 (isr1) and Ws-0 (isr1) as host plants.
\end{abstract}

Key words: Arabidopsis, basal resistance, ethylene, induced systemic resistance, plant defence, Pseudomonas fluorescens, systemic acquired resistance.

\author{
Abbreviations: \\ ISR: induced systemic resistance \\ SAR: $\quad$ systemic acquired resistance \\ SA: $\quad$ salicylic acid \\ JA: jasmonic acid \\ ET: ethylene
}

\section{Introduction}

Selected strains of non-pathogenic, root-colonizing bacteria are capable of inducing systemic resistance against a broad spectrum of pathogens. This phenomenon is commonly referred to as rhizobacteria-mediated ISR (Van Loon et al., $\left.1998^{[11]}\right)$. To study the molecular basis underlying rhizobacteria-mediated ISR, an Arabidopsis-based model system was

Plant biol. 4 (2002) 224-227

(c) Georg Thieme Verlag Stuttgart · New York ISSN 1435-8603 developed (Pieterse et al., 1996 ${ }^{[3]}, 2001^{[2]}$ ). Colonization of Arabidopsis roots by ISR-inducing $P$. fluorescens WCS417r bacteria protects the plant against diseases caused by different types of pathogens, including the bacterial leaf pathogens $P$. syringae pv. tomato and Xanthomonas campestris pv. armoraciae, the oomycetous leaf pathogen Peronospora parasitica, and the fungal pathogens Fusarium oxysporum f.sp. raphani and Alternaria brassicicola (Pieterse et al., 1996[3]; Ton et al., 2002 ${ }^{[10]}$ ). Rhizobacteria-mediated ISR resembles pathogen-induced SAR (Ryals et al., 1996[5]; Sticher et al., 1997[6]), in that it is effective against a range of different pathogens. However, in Arabidopsis the SAR and the ISR signalling pathways clearly diverge. SAR is regulated by a SA-dependent pathway and is associated with the co-ordinate expression of genes encoding pathogenesis-related (PR) proteins (Ryals et al., 1996 ${ }^{[5]}$ ). By contrast, WCS417r-mediated ISR is controlled by a JA- and ET-dependent pathway and is not associated with the activation of $P R$ genes (Pieterse et al., 1998 ${ }^{[4]}, 2001^{[2]}$ ). Also, the spectrum of effectiveness partly diverges. In contrast to SAR, ISR is effective against pathogens that, in non-induced plants, are resisted through JA/ ET-dependent basal defences, e.g., A. brassicicola. Conversely, SAR is effective against pathogens that in non-induced plants are resisted through SA-dependent defences, e.g., $P$. parasitica and turnip crinkle virus (TCV), whereas ISR is only weakly effective ( $P$. parasitica) or not effective at all (TCV) against these pathogens (Ton et al., 2002 ${ }^{[10]}$ ). Both ISR and SAR are effective against the bacterial pathogens $P$. syringae pv. tomato and $X$. campestris pv. armoraciae that in non-induced plants are resisted through a combination of SA-, JA- and ET-dependent basal defences (Pieterse et al., 1998 ${ }^{[4]}$; Ton et al., 2002 ${ }^{[10]}$ ). Interestingly, simultaneous activation of both types of induced resistance results in an enhanced level of protection against $P$. syringae pv. tomato, indicating that ISR and SAR can have an additive effect on the level of induced protection (Van Wees et al., 2000[13]).

The Arabidopsis accession Col-0, as well as most other accessions, express ISR against $P$. syringae pv. tomato after treatment of the roots with WCS417r. However, accessions RLD1 and Ws-0 fail to do so (Ton et al., 1999[8]; Van Wees et al., $\left.1997^{[12]}\right)$. Further characterization of several ISR-inducible accessions and the non-inducible accessions RLD1 and Ws-0 revealed that the inability to express WCS417r-mediated ISR against $P$. syringae pv. tomato is associated with an enhanced susceptibility to this pathogen. Genetic analysis of progeny of crosses between inducible and non-inducible Arabidopsis ac- 
cessions showed that inducibility of ISR and basal resistance against $P$. syringae pv. tomato are controlled by a single dominant locus (ISR1), that maps to chromosome III (Ton et al., $\left.1999^{[8]}\right)$. Interestingly, both the non-inducible accessions RLD1 and Ws-0 showed a reduced sensitivity to ET. This reduced sensitivity to ET co-segregated with the recessive alleles at the ISR 1 locus in the $\mathrm{F}_{2}$ progeny of a cross between Col-0 and RLD1 (Ton et al., 2001 ${ }^{[9]}$ ). Therefore, it was proposed that the Arabidopsis ISR1 locus encodes a novel component of the ET response pathway that plays an important role in disease resistance.

The dual involvement of the ISR1 locus in WCS417r-mediated ISR against $P$. syringae pv. tomato, on the one hand, and basal resistance against this pathogen, on the other hand, prompted us to investigate whether the ISR1 locus plays a similar role in both ISR and basal resistance against other pathogens. In a comparative study between accession Col-0, carrying the dominant alleles at the ISR1 locus, and accessions RLD1 and Ws- 0 , carrying the recessive isr 1 alleles, we investigated the role of the ISR1 locus in WCS417r-mediated ISR against the Arabidopsis pathogens $P$. syringae pv. tomato, X. campestris pv. armoraciae, $P$. parasitica and TCV. In addition, we examined the role of the ISR1 locus in SAR and basal resistance against these pathogens.

\section{Materials and Methods}

All bioassays were performed as described previously (Ton et al., 2002[10]). For experimental details see legend to Fig. 1.

\section{Results and Discussion}

To investigate the involvement of the ISR1 locus in basal resistance against $X$. campestris pv. armoraciae, $P$. parasitica and TCV, ISR1 plants (Col-0) were compared with isr1 plants (RLD1 or Ws-0) for their level of basal resistance against these pathogens. Consistent with previous findings (Ton et al., 1999 ${ }^{[8]}$ ), the isr1 genotype Ws-0 showed a higher disease severity after inoculation with P. syringae pv. tomato (Fig. 1 A), and allowed 5 -fold more growth of the pathogen in the non-induced leaves compared to the ISR1 genotype Col-0 (Fig. 1B). However, a higher rather than a lower level of basal resistance against $X$. campestris pv. armoraciae and $P$. parasitica was apparent in isr1 genotypes RLD1 and Ws-0, respectively. Three days after primary inoculation with $X$. campestris pv. armoraciae, control-treated Col-0 plants developed more bacterial spot disease (Fig. 1 C), and allowed higher levels of growth of the pathogen than control-treated RLD1 plants (Fig. 1D). Similarly, control-treated Col-0 plants were more susceptible to $P$. parasitica than control-treated Ws-0 plants, as was evident from a higher disease incidence (Fig. 1 E) and a two-fold higher production of sporangia by the pathogen (Fig. 1 F). For TCV, the disease severity in non-inoculated leaves of infected RLD1 plants was somewhat higher compared to that of similarly treated Col-0 plants (Fig. 1G). Nevertheless, the extent of viral multiplication in these plants was similar for both genotypes (Fig. 1 H), indicating that both the ISR 1 genotype Col-0 and the isr1 genotype RLD1 exhibit equal levels of susceptibility to TCV. Together, these results indicate that there is no consistent effect of the ISR1 locus on the level of basal resistance against $X$. campestris pv. armoraciae, P. parasitica and TCV.
Besides being involved in basal resistance against $P$. syringae pv. tomato, the ISR1 locus is also required for WCS417r-mediated ISR against this pathogen (Ton et al., 1999[8]). Indeed, ISR1 genotype Col-0, unlike isr1 genotype Ws-0, developed significantly less disease symptoms (Fig. 1 A) and allowed lower levels of growth of $P$. syringae pv. tomato (Fig. 1 B) after treatment of the roots with WCS417r. Similarly, disease symptoms caused by $X$. campestris pv. armoraciae, as well as growth of this bacterium in the leaves, were significantly reduced in ISR1 genotype Col-0 after treatment with WCS417r, whereas the isr1 genotype RLD1 failed to develop WCS417r-mediated ISR against this pathogen (Figs. 1C,D). Upon challenge with $P$. parasitica, the same situation applied. Only the ISR1 genotype Col-0 expressed WCS417r-mediated ISR, as was evident from a reduction in disease severity (Fig. 1E), and a 7.5-fold reduction of pathogen sporulation in WCS417r-treated plants (Fig.1F). The isr1 genotype Ws-0 failed to develop resistance against $P$. parasitica after treatment with WCS417r (Figs. 1E,F). These results demonstrate that the ISR1 locus is required for the expression of WCS417r-mediated ISR against these three pathogens. In contrast, neither the ISR1 genotype Col-0, nor the isr1 genotype RLD1 showed a reduction in viral disease symptoms caused by TCV after treatment of the roots with WCS417r (Fig. 1G). These results support our previous finding that WCS417r-mediated ISR is ineffective against TCV (Ton et al., $\left.2002^{[10]}\right)$.

To elucidate whether the ISR1 locus also influences the expression of SAR, ISR1 plants (Col-0) were compared with isr1 plants (RLD1 or Ws-0) for their ability to express SAR against the four pathogens. SAR was triggered either by predisposal infection of two lower leaves with avirulent $P$. syringae pv. tomato DC3000 carrying the avirulence gene avrRpt2, or by spraying the plants with the chemical SAR inducer 2,6-dichloroisonicotinic acid (INA). After induction of SAR, both the ISR1 genotype Col-0 and the isr1 genotypes RLD1 and Ws-0 showed a significant reduction in disease symptoms and bacterial proliferation after challenge inoculation with virulent $P$. syringae pv. tomato and $X$. campestris pv. armoraciae (Figs.1A-D). Similarly, induction of SAR against $P$. parasitica and TCV was equally effective in ISR1 genotype Col-0 and isr1 genotypes Ws-0 and RLD1 (Figs.1E-G). It can thus be concluded that the ISR1 locus is not involved in the expression of SAR against these different pathogens.

In conclusion, we showed that the ISR1 locus does not contribute to basal resistance against $X$. campestris pv. armoraciae, $P$. parasitica and TCV. Recently, we demonstrated that the isr1 genotypes RLD1 and Ws-0 exhibit reduced responsiveness to ET, indicating that the ISR1 locus is involved in ET signalling (Ton et al., 2001 ${ }^{[9]}$ ). Thus, in contrast to its role in basal resistance against $P$. syringae pv. tomato, ET signalling is of less relevance to basal resistance against $X$. campestris pv. armoraciae, P. parasitica and TCV. Accordingly, Thomma et al. $\left(1998^{[7]}\right)$ reported that basal resistance against $P$. parasitica is unaffected in the ET-insensitive mutant ein2-1, whereas Kachroo et al. $\left(2000^{[1]}\right)$ demonstrated that the ein2-1 mutation does not confer enhanced susceptibility to TCV. However, ein2-1 plants did allow higher levels of growth of $X$. campestris pv. armoraciae in the leaves compared to wild-type Col-0 plants (Ton et al., $2001 \mathrm{a}^{[9]}$ ). These observations suggest that additional genetic differences between isr1 genotype RLD1 and ISR1 genotype 
A

PI:

$m$

F. purasilite

TCH

C

$\mathbf{G}$
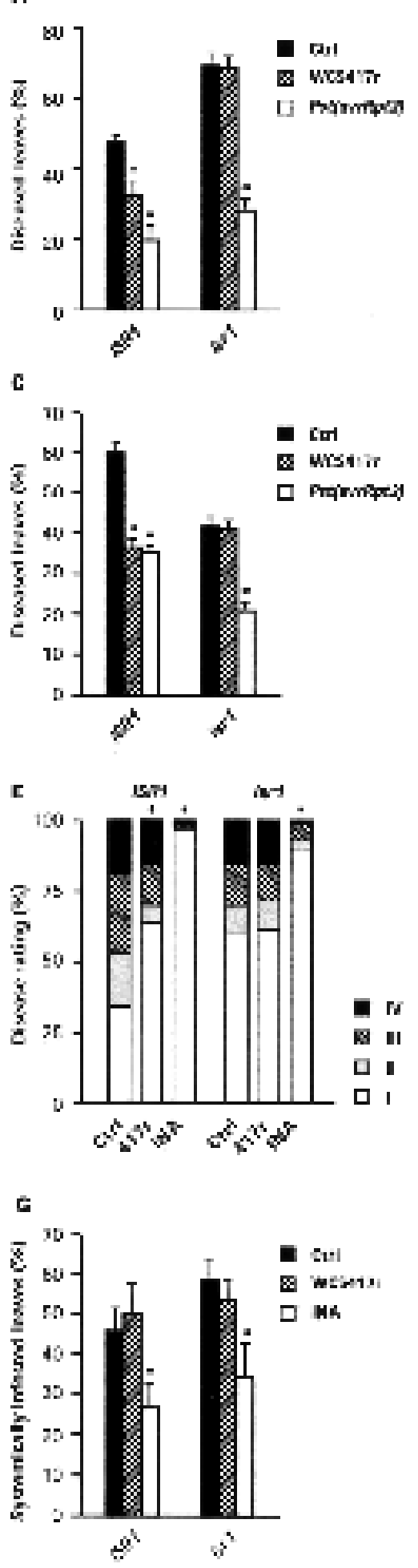

E

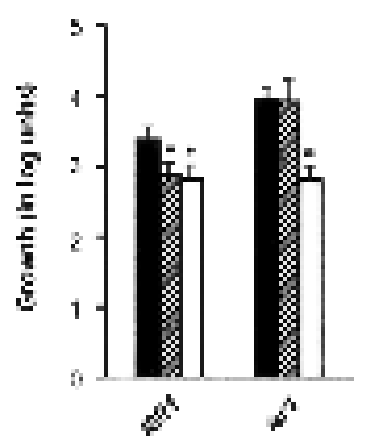

口

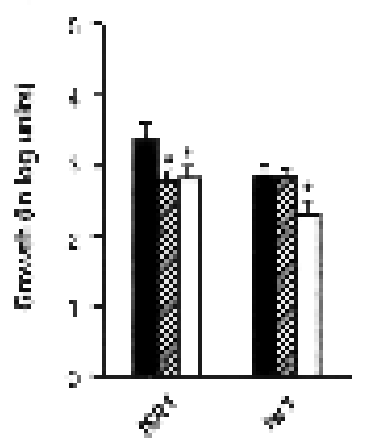

F

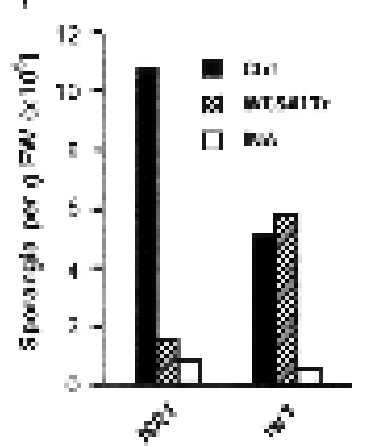

H

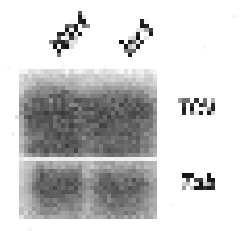

Fig.1 Quantification of basal and induced resistance against $P$. syringae pv. tomato DC3000 (A, B) X. campestris pv. armoraciae $(\mathbf{C}, \mathbf{D})$ P. parasitica $(\mathbf{E}, \mathbf{F})$ and TCV $(\mathbf{G}, \mathbf{H})$ in ISR1 and isr1 plants. Bioassays were performed essentially as described (Ton et al., $2002^{[10]}$. Differences in basal resistance are reflected by the differences in disease severity and pathogen proliferation in non-induced control plants (Ctrl). ISR was induced by treatment of the roots with $P$. fluorescens WCS417r bacteria. SAR was induced three days prior to challenge inoculation, either by infection of two lower leaves with $P$. syringae pv. tomato DC3000 (avrRpt2), or by spraying the leaves with INA. For $P$. syringae pv. tomato DC3000 and X. campestris pv. armoraciae bioassays, 5-week-old plants were challenged by dipping the leaves in a bacterial suspension containing $2.5 \times 10^{7} \mathrm{CFU}_{\mathrm{ml}}{ }^{-1}$ or $5 \times 10^{7} \mathrm{CFU} \cdot \mathrm{ml}^{-1}$, respectively. The percentage of leaves with symptoms $(n=20)(\mathbf{A}, \mathbf{C})$ and bacterial proliferation $(n=5)(B, D)$ were determined 3 days after challenge. Leaves showing necrotic or water-soaked lesions surrounded by chlorosis were scored as diseased. For $P$. parasitica bioassays, 3-weekold plants were challenged by applying $3-\mu l$ droplets containing $5 \times 10^{4}$ conidiospores per $\mathrm{ml}$. Disease symptoms (E), and sporangia production (F) were determined 11 days after challenge. Disease rating was expressed as percentage of leaves $(n=200)$ in disease severity classes: I, no sporulation; II, $<50 \%$ of the leaf area covered by sporangia; III, $>50 \%$ of the leaf area covered by sporangia; IV, leaves heavily covered with sporangia, with additional chlorosis and leaf collapse. For TCV bioassays, 4-week-old plants were challenged by rubbing $3 \mu$ droplets of viral RNA suspension $\left(0.1 \mu \mathrm{g} \cdot \mu^{-1}\right)$ in bentonite buffer onto three lower leaves. At 14 days after challenge, the percentage of systemically infected leaves with symptoms was determined per treatment $(n=20)(\mathbf{G})$, and 5 representative control-treated plants were harvested for RNA blot analysis $(\mathbf{H})$. Systemically infected leaves showing crinkled deformation of the leaves and chlorotic spots around the vascular bundles were scored as diseased. Error bars indicate standard errors of the mean. Asterisks indicate statistically significant differences between induction and control treatments, according to the Student's t-test $(\mathbf{A}-\mathbf{D}, \mathbf{G})$, or the Chisquare test $(\mathbf{E})(\alpha=0.05)$. ISR1: accession Col-0; isr1: accession RLD1 $(\mathbf{C}, \mathbf{D}, \mathbf{G}, \mathbf{H})$ or Ws-0 (A, B, E, F).
Col-0 influence the level of basal resistance against $X$. campestris pv. armoraciae.

Furthermore, we showed that the ISR1 locus is not only involved in WCS417r-mediated ISR against P. syringae pv. tomato but also in ISR against $X$. campestris pv. armoraciae and $P$. para- sitica. Similar results were reported for the fungal pathogen F. oxysporum f.sp. raphani by Van Wees et al. (1997 $\left.{ }^{[12]}\right)$, who showed that, in contrast to Col-0, RLD1 is unable to express WCS417r-mediated ISR against this pathogen. Taken together, these results clearly demonstrate that the ISR1 locus plays a key role in WCS417r-mediated ISR against various plant patho- 
gens. Because the ISR1 locus is involved in ET signalling (Ton et al., 2001 ${ }^{[9]}$ ), this indicates that intact responsiveness to ET is an important prerequisite for the broad-spectrum resistance conferred by WCS417r-mediated ISR.

\section{Acknowledgements}

We thank Ientse van der Sluis for technical assistance and Drs. Andrew Bent, Mark Aarts, Sarah Miller, and Anne Simon, for kindly providing isolates of $P$. syringae pv. tomato, $P$. parasitica, $X$. campestris pv. armoraciae and TCV, respectively.

\section{References}

${ }^{1}$ Kachroo, P., Yoshioka, K., Shah, J., Dooner, H. K., and Klessig, D. F. (2000) Resistance to turnip crinkle virus in Arabidopsis is regulated by two host genes and is salicylic acid dependent but NPR1, ethylene and jasmonate independent. Plant Cell 12, 677-690.

2 Pieterse, C. M. J., Van Pelt, J. A., Van Wees, S. C. M., Ton, J., LéonKloosterziel, K. M., Keurentjes, J. J. B., Verhagen, B. W. M., Knoester, M., Van der Sluis, I., Bakker, P. A. H. M., and Van Loon, L. C. (2001) Rhizobacteria-mediated induced systemic resistance: triggering, signalling and expression. Eur. J. Plant Pathol. 107, 51 - 61.

${ }^{3}$ Pieterse, C. M. J., Van Wees, S. C. M., Hoffland, E., Van Pelt, J. A., and Van Loon, L. C. (1996) Systemic resistance in Arabidopsis induced by biocontrol bacteria is independent of salicylic acid accumulation and pathogenesis-related gene expression. Plant Cell 8, $1225-1237$.

${ }^{4}$ Pieterse, C. M. J., Van Wees, S. C. M., Van Pelt, J. A., Knoester, M., Laan, R., Gerrits, H., Weisbeek, P. J., and Van Loon, L. C. (1998) A novel signaling pathway controlling induced systemic resistance in Arabidopsis. Plant Cell 10, 1571-1580.

${ }^{5}$ Ryals, J. A., Neuenschwander, U. H., Willits, M. G., Molina, A., Steiner, H., and Hunt, M. D. (1996) Systemic acquired resistance. Plant Cell 8, 1809-1819.

${ }^{6}$ Sticher, L., Mauch-Mani, B., and Métraux, J.-P. (1997) Systemic acquired resistance. Annu. Rev. Phytopathol. 35, 235-270.

${ }^{7}$ Thomma, B. P. H. J., Eggermont, K., Penninckx, I. A. M. A., MauchMani, B., Vogelsang, R., Cammue, B. P. A., and Broekaert, W. F. (1998) Separate jasmonate-dependent and salicylate-dependent defense-response pathways in Arabidopsis are essential for resistance to distinct microbial pathogens. Proc. Natl. Acad. Sci. USA 95, $15107-15111$

${ }^{8}$ Ton, J., Pieterse, C. M. J., and Van Loon, L. C. (1999) Identification of a locus in Arabidopsis controlling both the expression of rhizobacteria-mediated induced systemic resistance (ISR) and basal resistance against Pseudomonas syringae pv. tomato. Mol. Plant-Microbe Interact. 12, $911-918$.

${ }^{9}$ Ton, J., Davison, S., Van Wees, S. C. M., Van Loon, L. C., and Pieterse, C. M. J. (2001) The ISR1 locus of Arabidopsis controlling induced systemic resistance is involved in ethylene signaling. Plant Physiol. $125,652-661$.

${ }^{10}$ Ton, J., Van Pelt, J. A., Van Loon, L. C., and Pieterse, C. M. J. (2002) Differential effectiveness of salicylate-dependent, and jasmonateand ethylene-dependent induced resistance in Arabidopsis. Mol. Plant-Microbe Interact. 15, 27 - 34.

11 Van Loon, L. C., Bakker, P. A. H. M., and Pieterse, C. M. J. (1998) Systemic resistance induced by rhizosphere bacteria. Annu. Rev. Phytopathol. 36, 453-485.

12 Van Wees, S. C. M., Pieterse, C. M. J., Trijssenaar, A., Van't Westende, Y. A. M., Hartog, F., and Van Loon, L. C. (1997) Differential induction of systemic resistance in Arabidopsis by biocontrol bacteria. Mol. Plant-Microbe Interact. 10, 716- 724.
${ }^{13}$ Van Wees, S. C. M., De Swart, E. A. M., Van Pelt, J. A., Van Loon, L. C., and Pieterse, C. M. J. (2000) Enhancement of induced disease resistance by simultaneous activation of salicylate- and jasmonatedependent defense pathways in Arabidopsis thaliana. Proc. Natl. Acad. Sci. USA 95, 1933-1937.

\author{
C. M. J. Pieterse \\ Phytopathology \\ Faculty of Biology \\ Utrecht University \\ P.O. Box 800.84 \\ 3508 TB Utrecht \\ The Netherlands \\ E-mail: c.m.j.pieterse@bio.uu.nl \\ Section Editor: J. Draper
}

